\title{
Clinical features and diagnosis of small-vessel vasculitis
}

\author{
CAROL LANGFORD, MD, MHS
}

Director, Center for Vasculitis Care and Research, Department of Rheumatic and Immunologic Disease, Cleveland Clinic, Cleveland, $\mathrm{OH}$

\section{ABSTRACT}

Vasculitis is inflammation of the blood vessel. Granulomatosis with polyangiitis (GPA), microscopic polyangiitis, and eosinophilic GPA are three small-vessel vasculitic diseases that share certain features, but also have important differences. Distinguishing these entities may influence the diagnostic approach, treatment decisions, and outcomes. Circulating antineutrophil cytoplasmic antibodies (ANCA) characterize all three diseases, although their immunofluorescence patterns and target antigen specificities differ. While the presence of ANCA can suggest these diagnoses, the diseases are best viewed as separate entities, each defined by specific clinical and histologic characteristics.

$\mathbf{V}$ asculitis refers to inflammation of the blood vessel. This inflammation can cause vessel wall thickening that compromises or occludes the vessel lumen, ultimately resulting in organ ischemia. It also can cause vessel wall attenuation that predisposes to aneurysm formation or breach of the vessel integrity with resultant hemorrhage into the tissue.

Vasculitis can be thought of as a primary or secondary process. Primary vasculitides are unique disease entities without a currently identified underlying cause in which vasculitis forms the pathologic basis of tissue injury. Vasculitis can occur secondary to medication exposure or an underlying illness, including infections, malignancy, cryoglobulinemia, and rheumatic diseases (such as systemic lupus erythematosus, rheumatoid arthritis, Sjögren syndrome, or myositis).

Primary vasculitides may differ in epidemiology, such as the age at which they occur and the gender most likely to be affected, their clinical manifestations (including signs, symptoms, and patterns of organ involvement), the diagnostic approach (biopsy, arteriography, and laboratory investigation), treatment (supportive care, glucocorticoids alone, or in combination with other immunosuppressants), and the size of the vessels predominantly affected (large, medium, or small).

Small-vessel vasculitis affects the arteriole, capillary, and venule. An excellent example of small-vessel vasculitis and the one most commonly encountered in clinical practice is cutaneous vasculitis, in which extravasation

Dr. Langford reported that she has no financial interests or relationships that pose a potential conflict of interest with this article.

This article was developed from an audio transcript of Dr. Langford's presentation at the "New Directions in Small-Vessel Vasculitis: ANCA, Target Organs, Treatment, and Beyond" symposium held at Cleveland Clinic on May 4, 2011. of erythrocytes from disrupted small vessels is observed histologically, with the clinical sequelae of palpable purpura. Although categorization based on the predominant vessel size that is affected is a helpful way to view these diseases, this is not absolute and each disease has the potential to affect a diverse range of vessels.

This article explores the clinical features and diagnosis of three forms of vasculitis that predominantly affect the small vessels: granulomatosis with polyangiitis (GPA [Wegener's granulomatosis]), microscopic polyangiitis (MPA), and eosinophilic GPA (Churg-Strauss syndrome, EGPA).

\section{GRANULOMATOSIS WITH POLYANGIITIS}

Granulomatosis with polyangiitis is characterized by granulomatous inflammation involving the respiratory tract and by vasculitis affecting small- to medium-sized vessels in which necrotizing glomerulonephritis is common.

\section{Wide range of presentations, manifestations}

Approximately $90 \%$ of patients with GPA have upper or lower airway involvement or both. ${ }^{1}$ Upper airway or ear symptoms affect $73 \%$ of patients initially and $92 \%$ overall. ${ }^{1}$ Direct inspection of the nasal membranes shows a cobblestoned or ulcerated appearance, and computed tomography reveals mucosal thickening of the sinuses. In some instances, sinus disease can compromise blood supply to the cartilaginous portion of the nasal septum, leading to nasal septum perforations or collapse of the nasal bridge. Another manifestation of upper airway disease and GPA is subglottic stenosis, a narrowing in the subglottic region located just below the vocal cords. The narrowing typically spans about $1 \mathrm{~cm}$ and rarely extends or involves the remainder of the trachea.

The lungs are involved in $85 \%$ of patients. ${ }^{1}$ Radiographic abnormalities can be diverse and include bilateral pulmonary nodular infiltrates, single or multiple cavities, and bilateral ground glass infiltrates as can be seen in pulmonary hemorrhage (Figure). Bronchoscopy may reveal endobronchial stenosis, and pleural disease can occur rarely.

Approximately 20\% of patients with GPA may have glomerulonephritis when they first present for medical attention, but it eventually develops in nearly $80 \%$ of patients during the disease course. ${ }^{1}$ Despite its potential

The transcript was formatted and edited by Cleveland Clinic Journal of Medicine staff for clarity and conciseness, and was then reviewed, revised, and approved by Dr. Langford.

doi:10.3949/ccjm.79.s3.01 

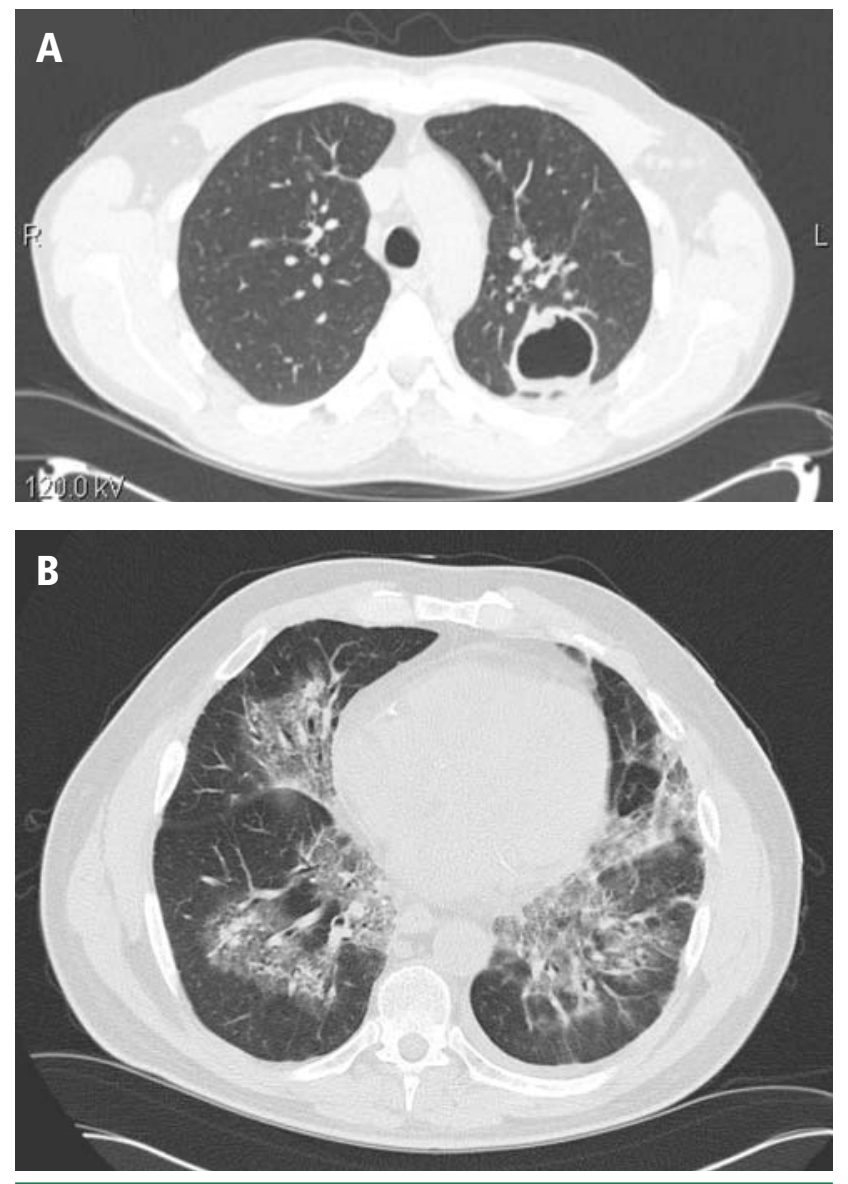

FIGURE. Computed tomography demonstrating two radiographic presentations of granulomatosis with polyangiitis (Wegener's granulomatosis): (A) cavitary lung disease; (B) bilateral ground glass infiltrates in a patient with alveolar hemorrhage.

for rapid progression, glomerulonephritis presents a diagnostic challenge because it is asymptomatic. It is detected by evidence of proteinuria and an active urine sediment with dysmorphic red blood cells and red blood cell casts.

Ocular involvement occurs eventually in 52\% of patients with GPA. ${ }^{1}$ Any ocular structure can be affected and ocular involvement can be visually threatening. The more prominent ocular manifestations include scleritis/episcleritis or orbital disease.

Cutaneous manifestations, observed in $46 \%$ of patients, include verrucous-appearing lesions on the elbow and infarctions in the skin and nail folds. ${ }^{1}$ Other rare manifestations can occur, such as pericarditis and cerebral vasculitis.

Although nearly all patients present with upper or lower airway symptoms, the multisystem nature of GPA explains the wide range of presentations and the varying degrees of disease severity.

\section{Differential diagnosis}

The differential diagnosis in GPA is varied. Particularly in the setting of isolated lung or sinus disease, infection is foremost in the differential diagnosis. Even in the nonimmunosuppressed host, unusual infections such as mycobacteria, histoplasmosis, and other fungal infections should be considered. Lymphadenopathy, rarely seen in GPA, should raise concern for other causes of disease. Lymphoproliferative processes and other neoplasms, other rheumatic diseases, granulomatous disease (ie, sarcoidosis), and other causes of glomerulonephritis (when present) also merit consideration. Differentiation of these entities from GPA is essential because the treatment differs in many instances.

The differential diagnosis for patients who present with midline destructive lesions must include other causes of collapse of the nasal bridge, nasal septum perforation, and possibly palate destruction. Erosions of the hard palate in particular should raise an immediate red flag for entities other than GPA, such as lymphoproliferative diseases; rare infections, particularly if the patient has studied or worked abroad; and cocaine exposure.

\section{Diagnostic evaluation}

A diagnosis of GPA is typically based on the presence of histologic features in a clinically compatible setting. Diagnostic features include necrosis, granulomatous inflammation, vasculitis, and special stains and cultures negative for microorganisms.

Biopsy sites are determined by evidence of clinical disease affecting a target organ and the likelihood of obtaining diagnostically meaningful findings from that site. One challenge is that biopsies are not always diagnostic. The changes tend to be patchy and the likelihood of a positive yield is associated with the amount of tissue that can be obtained. Tissues from the ear, nose, and throat have a yield of about $20 \%$, depending upon the site and the biopsy size. The highest yield comes from radiographically abnormal pulmonary parenchyma. Although transbronchial biopsies are attractive because they are less invasive than open lung biopsy, they are also far less diagnostic, with fewer than 10\% having a positive yield. Because cutaneous vasculitis is observed in many settings, its presence is usually insufficient evidence for diagnosis. The renal histologic appearance is a focal, segmental, crescentic, and necrotizing glomerulonephritis that has few to no immune complexes (pauciimmune glomerulonephritis). ${ }^{1-3}$

Chest imaging should be performed in any patient in whom GPA is part of the differential diagnosis, since up to one-third of patients may be asymptomatic yet have pulmonary radiographic findings.

Laboratory assessment should include serum chemistries to evaluate renal and hepatic function, complete blood count, erythrocyte sedimentation rate, measurement of $\mathrm{C}$-reactive protein, and urinalysis. If the urinalysis is positive for blood, microscopy should be performed on fresh urine to look for casts. In the setting of pulmonary-renal manifestations, testing for other 
causes, such as antiglomerular basement antibodies and antinuclear antibodies, should be considered.

Serologic testing for antineutrophil cytoplasmic antibodies (ANCA) has provided a useful tool in suggesting the diagnosis of GPA. Two forms of ANCA have been identified in patients with vasculitis: ANCA directed against the neutrophil serine protease proteinase-3 (PR3), which results in a cytoplasmic immunofluorescence (cANCA) pattern; and ANCA directed against the neutrophil enzyme myeloperoxidase (MPO), which causes a perinuclear immunofluorescence (pANCA) pattern. ${ }^{4}$ Approximately $80 \%$ to $95 \%$ of ANCA found in patients with active severe GPA are detectable PR3cANCA, while $5 \%$ to $20 \%$ are MPO-pANCA..$^{5}$ The predictive value of ANCA for the diagnosis depends on the spectrum of clinical features. As ANCA can be seen in other settings, ANCA as the basis for diagnosis in place of tissue biopsy should be used with caution and only in selected instances where their predictive value would equal that of biopsy. The presence of ANCA is not necessary to establish the diagnosis, as up to $20 \%$ of patients with GPA may be ANCA-negative. ${ }^{6}$

\section{MICROSCOPIC POLYANGIITIS}

The history of MPA dates to 1866, with the description of periarteritis nodosa. ${ }^{7}$ The term "microscopic polyarteritis" was introduced in 1948, when glomerular disease was recognized in some patients. ${ }^{8}$ In 1994, the Chapel Hill Consensus Conference defined MPA as a necrotizing vasculitis with few or no immune deposits that affects small vessels (ie, capillaries, venules, or arterioles). Necrotizing arteritis of small- and medium-sized arteries may be present. Necrotizing glomerulonephritis and pulmonary capillaritis commonly occur. ${ }^{9}$ MPA shares many clinical features with GPA and is currently said to be distinguished by the absence of granulomatous inflammation. ${ }^{9}$

\section{Presentations and manifestations}

In one assessment of organ system involvement in 85 patients with MPA, investigators observed glomerular syndrome in $82 \%$ of patients. ${ }^{10}$ They also found a high predilection for involvement of the skin, joints, and lungs. Pulmonary hemorrhage is a particularly important manifestation of MPA because it can be immediately life-threatening.

\section{Differential diagnosis}

The differential diagnosis for MPA is similar to GPA in the inclusion of other causes of classic pulmonary-renal syndromes, such as antiglomerular basement membrane disease and systemic lupus erythematosus. Poststreptococcal glomerulonephritis should be considered when the kidney is the predominant organ involved in the absence of lung disease. In the setting of pulmonary infiltrates, infections and neoplasms remain significant in the differential diagnosis.

\section{Diagnostic evaluation}

The diagnosis of MPA is based on consistent clinical features and compatible histologic findings. The histologic renal lesion is identical to that seen in GPA. Pulmonary disease typically includes capillaritis and is notable for the absence of evidence of immune deposition, in contrast to antiglomerular basement membrane disease.

Chest imaging is indicated when MPA is part of the differential diagnosis. Computed tomography is the preferred technique, as early alveolar hemorrhage that can occur in MPA may not be visualized on a chest radiograph.

Laboratory assessment should include serum chemistries, complete blood count, erythrocyte sedimentation rate, measurement of $\mathrm{C}$-reactive protein, and urinalysis. Additional testing should be pursued for other diseases as indicated by the clinical features.

Approximately $40 \%$ to $80 \%$ of patients with MPA have MPO-pANCA. ${ }^{5}$ Approximately $15 \%$ of patients are MPO-pANCA positive, ${ }^{6}$ and $0 \%$ to $20 \%$ are ANCA-negative. As with GPA, ANCA is useful to suggest-but not diagnose-disease in many instances. The absence of ANCA does not rule out MPA.

\section{EOSINOPHILIC GPA}

Eosinophilic GPA is a unique entity characterized by eosinophil-rich and granulomatous inflammation involving the respiratory tract and necrotizing vasculitis of small- to medium-size vessels. It is also associated with asthma and eosinophilia.

\section{Different disease phases}

Eosinophilic GPA is often thought of as having three phases: prodromal, eosinophilic, and vasculitic. ${ }^{11,12}$ Although helpful conceptually, these phases may not always be present and may not occur in sequence.

The prodromal phase is characterized by asthma associated with allergic rhinitis with or without polyposis. The eosinophilic phase is characterized by the presence of eosinophilia in the blood and tissue. Eosinophilia is a prominent feature, although accurate detection and assessment can be challenging in the setting of glucocorticoid use for asthma as this normalizes the eosinophil count.

The vasculitic phase distinguishes EGPA from other eosinophilic disorders. Features of vasculitis may occur in multiple organ sites, including the nerves, lungs, heart, gastrointestinal tract, and kidneys. In one series of 96 patients, nearly 100\% had asthma, and peripheral nervous system involvement in the form of mononeuritis multiplex was present in $72 \% .{ }^{12}$ Cardiac involvement is of particular importance as it is a prominent cause of disease-related mortality. Cardiac manifestations include myocarditis, pericarditis, endocarditis, valvulitis, and coronary vasculitis. 
TABLE

Differential diagnosis

\begin{tabular}{|c|c|c|c|}
\hline & $\begin{array}{l}\text { Granulomatosis } \\
\text { with } \\
\text { polyangiitis (GPA) }\end{array}$ & $\begin{array}{l}\text { Microscopic } \\
\text { polyangiitis }\end{array}$ & $\begin{array}{c}\text { Eosinophilic } \\
\text { GPA }\end{array}$ \\
\hline $\begin{array}{l}\text { Ear, nose, } \\
\text { throat }\end{array}$ & $\begin{array}{l}\text { Necrotizing, } \\
\text { destructive }\end{array}$ & - & Allergic \\
\hline Lung & $\begin{array}{l}\text { Nodule, cavity, } \\
\text { infiltrate }\end{array}$ & Infiltrates & $\begin{array}{l}\text { Asthma, } \\
\text { infiltrates, } \\
\text { nodule }\end{array}$ \\
\hline Kidney & ++++ & ++++ & +-++ \\
\hline Nerve & ++ & +++ & ++++ \\
\hline Skin & ++ & +++ & +++ \\
\hline Heart & + & + & ++ (mortality) \\
\hline Granuloma & ++++ & - & ++++ \\
\hline Eosinophils & - & - & ++++ \\
\hline ANCA & $\begin{array}{c}80 \%-95 \% \text { PR3 } \\
5 \%-20 \% \text { MPO } \\
0 \%-20 \% \text { ANCA (-) }\end{array}$ & $\begin{array}{c}40 \%-80 \% \text { MPO } \\
35 \% \text { PR3 } \\
0 \%-20 \% \text { ANCA }(-)\end{array}$ & $\begin{array}{c}40 \% \text { MPO } \\
35 \% \text { PR3 } \\
\text { Up to } 60 \% \text { ANCA (-) }\end{array}$ \\
\hline
\end{tabular}

ANCA = antineutrophil cytoplasmic antibodies; $\mathrm{MPO}=$ myeloperoxidase; $\mathrm{PR3}=$ proteinase -3

$-=$ absent $+=$ relative frequency

\section{Differential diagnosis}

The differential diagnosis of EGPA shares similarities with GPA and MPA, but also includes eosinophilic disorders such as hypereosinophilic syndrome, eosinophilic leukemia, and parasitic diseases.

\section{Diagnostic evaluation}

Diagnosis is often based on the presence of asthma, a finding of peripheral eosinophilia $\left(>1,500\right.$ cells $\left./ \mathrm{mm}^{3}\right)$, and the presence of systemic vasculitis involving, ideally, two or more extrapulmonary organs. While histologic confirmation remains ideal, demonstration of characteristic findings on biopsy can be difficult. Glomerular involvement is far less common than in GPA and MPA, but, when present, the renal lesion is identical. Pulmonary histologic findings can be diverse and include the classic "allergic-granuloma" as originally described by Churg and Strauss, as well as isolated granulomatous inflammation, eosinophilic inflammation, or smallvessel vasculitis. Tissue eosinophilia is a prominent finding that typically is seen on biopsies of skin, nerve, and gastrointestinal tissues.

Chest imaging should be performed when EGPA is part of the differential diagnosis. Because of the potential for cardiac involvement, a baseline echocardiogram should be obtained. Pulmonary function tests may be useful, particularly in patients who have a strong asthmatic component.

Similar to GPA and MPA, laboratory assessment includes serum chemistries, complete blood count with differential to determine the eosinophil count, erythrocyte sedimentation rate, measurement of C-reactive protein, and urinalysis. With the allergic and asthmatic components, immunoglobulin E levels are frequently elevated. Additional testing for other eosinophilic diseases should be pursued as indicated by the clinical features.

Only about $40 \%$ of patients are ANCApositive. ${ }^{13}$ Most of these are MPO-pANCA, with PR3-cANCA occurring less commonly. Although some reports have suggested differing clinical patterns of EGPA based on ANCA positivity, the presence or absence of ANCA is less helpful in the diagnosis. ${ }^{13}$

\section{DIFFERENTIATION}

Despite similarities, GPA, MPA, and EGPA are phenotypically unique. Because of differences in management, relapse risk, and outcome, differentiation is important. Several features can help distinguish these three small-vessel vasculitic diseases (Table). For example, upper airway disease, which tends to be necrotizing and destructive in GPA, is allergic in EGPA and absent in MPA. Lung disease in MPA tends to be pulmonary hemorrhage, which also can be seen in GPA. In GPA, however, nodular disease that may be cavitary is more common. Asthma is the predominant pulmonary feature in EGPA, although parenchymal nodules and hemorrhage also can be seen. While glomerulonephritis is typical in GPA and MPA, it occurs to a much lesser degree in EGPA. Cardiac features have particular importance in EGPA.

A key histologic difference between GPA and MPA is the presence of granulomatous inflammation in GPA and its absence in MPA under the current nomenclature system. ${ }^{9}$ Granulomatous inflammation can be seen in EGPA, but it is usually accompanied by eosinophils, which are less likely to be present in GPA and MPA.

The predominance of the ANCA immunofluorescence pattern and target antigen differs between GPA and MPA, with ANCA positivity occurring in 38\% of patients with EGPA. ${ }^{13}$

\section{SUMMARY}

Conceptualizing vasculitic disease based on vessel size can be useful, but it is not an absolute definition. Although GPA, MPA, and EGPA predominantly affect small- to medium-sized vessels, these disease entities are phenotypically unique, with both shared features and differences. Common to all three entities is the potential for organ- and life-threatening manifestations, particularly involving the lungs, kidneys, nerves, 
gastrointestinal tract, and heart. All three entities need aggressive immunosuppression for severe disease. Recognition of these entities and the distinctions among them can guide the approach to diagnosis, treatment, and future outcomes.

\section{REFERENCES}

1. Hoffman GS, Kerr GS, Leavitt RY, et al. Wegener granulomatosis: an analysis of 158 patients. Ann Intern Med 1992; 116:488-498.

2. Travis WD, Hoffman GS, Leavitt RY, Pass HI, Fauci AS. Surgical pathology of the lung in Wegener's granulomatosis: review of 87 open lung biopsies from 67 patients. Am J Surg Pathol 1991; 15:315-333.

3. Devaney KO, Travis WD, Hoffman G, Leavitt R, Lebovics R, Fauci AS. Interpretation of head and neck biopsies in Wegener's granulomatosis: a pathologic study of 126 biopsies in 70 patients. Am J Surg Pathol 1990; 14:555-564.

4. Bosch X, Guilabert A, Font J. Antineutrophil cytoplasmic antibodies. Lancet 2006; 368:404-418.

5. Hoffman GS, Specks U. Antineutrophil cytoplasmic antibodies. Arthritis Rheum 1998; 1521-1537.

6. Wiik A. What you should know about PR3-ANCA. An introduction. Arthritis Res 2000; 2:252-254.
7. Kussmaul A, Maier R. Über eine bisher nicht beschriebene eigenthümliche Arterienerkrankung (Periarteritis nodosa), die mit Morbus Brightii und rapid fortschreitender allgemeiner Muskellähmung einhergeht. Dtsch Arch Klin Med 1866; 1:484-518.

8. Davson J, Ball J, Platt R. The kidney in periarteritis nodosa. Q J Med 1948; 17:175-202.

9. Jennette C, Falk RJ, Andrassy K, et al. Nomenclature of systemic vasculitides: proposal of an international consensus conference. Arthritis Rheum 1994; 37:187-192.

10. Guillevin L, Durand-Gasselin B, Cevallos R, et al. Microscopic polyangiitis: clinical and laboratory findings in eighty-five patients. Arthritis Rheum 1999; 42:421-430.

11. Keogh KA, Specks U. Churg-Strauss syndrome. Semin Respir Crit Care Med 2006; 27:148-157.

12. Guillevin L, Cohen P, Gayraud M, Lhote F, Jarrousse B, Casassus P. Churg-Strauss syndrome: clinical study and long-term follow-up of 96 patients. Medicine 1999; 78:26-37.

13. Sablé-Fourtassou R, Cohen P, Mahr A, et al; for the French Vasculitis Study Group. Antineutrophil cytoplasmic antibodies and the Churg-Strauss syndrome. Ann Intern Med 2005; 143:632-638.

Correspondence: Carol Langford, MD, MHS, Department of Rheumatic and Immunologic Disease, Cleveland Clinic, 9500 Euclid Ave., A50, Cleveland, $\mathrm{OH}$ 44195; langfoc@ccf.org

\title{
Controversies in ANCA testing
}

\author{
ULRICH SPECKS, MD
}

Professor of Medicine, Division of Pulmonary and Critical Care Medicine, Mayo Clinic College of Medicine, Rochester, MN

\section{ABSTRACT}

Antineutrophil cytoplasmic antibody (ANCA) detection is a well-known tool for diagnosing small-vessel vasculitis. Its diagnostic utility, however, depends on the methodologic accuracy of the test and the appropriate ordering of testing in the right clinical setting. While ANCA testing is of proven value, the utility of serial ANCA testing is not entirely clear. Correlation of ANCA levels with disease activity and predicted relapse remains unconfirmed. The best gauge of the predictive value of serial testing is to perform long-term serial testing for some individual patients in order to establish a relationship between ANCA level and clinical disease manifestation over time. ANCA-antigen specificity can be used to assess prognosis in patients with ANCA-associated vasculitis. Proteinase 3-ANCA is associated with higher mortality, higher relapse rate, and faster renal deterioration compared with myeloperoxidase-ANCA. Overall, ANCA is an important diagnostic and prognostic marker for smallvessel vasculitis and warrants further investigation.

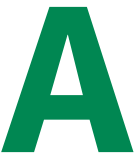
ntineutrophil cytoplasmic antibody (ANCA) detection is a valuable tool for diagnosing small-vessel vasculitis, ${ }^{1}$ but measuring and interpreting ANCA levels is an inexact science. There is no single perfect ANCA test, and even a perfect test would not provide definitive clinical answers. The diagnostic utility of ANCA testing depends on the methodologic accuracy of the test and the appropriate ordering of testing in the right clinical setting. This article examines three important questions about this technology:

- What is the best ANCA test methodology?

- What is the prognostic value of serial ANCA testing?

- What is the clinical implication of ANCA type?

\section{WHAT IS THE BEST ANCA TEST METHODOLOGY?}

The diagnostic utility of ANCA testing depends on both the methodologic accuracy of the test and the appropriate ordering of tests. Methodologic accuracy comprises
Dr. Specks reported that he has received consulting fees from Dynavax and Sanofi-Aventis.

Acknowledgment. Genentech provided drug and funding to the National Institute of Allergy and Infectious Disease for the conduct of the Rituximab in ANCA-Associated Vasculitis (RAVE) trial.

This article was developed from an audio transcript of Dr. Specks's presentation at the "New Directions in Small-Vessel Vasculitis: ANCA, Target Organs, Treatment, and Beyond" symposium held at Cleveland Clinic on May 4, 2011. The transcript was formatted and edited by Cleveland Clinic Journal of Medicine staff for clarity and conciseness, and was then reviewed, revised, and approved by Dr. Specks.

doi:10.3949/ccjm.79.s3.02 


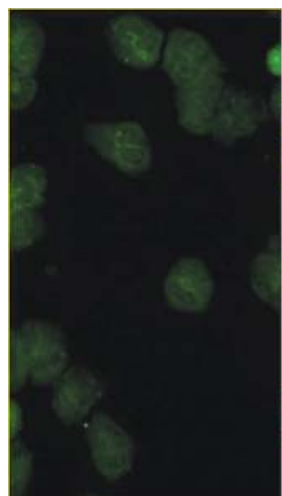

Normal

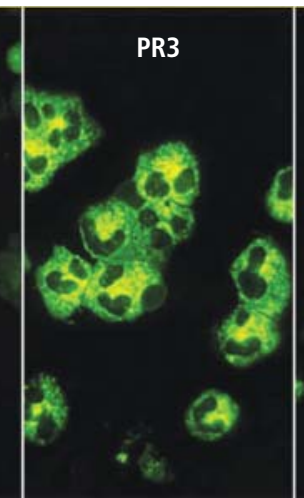

CANCA

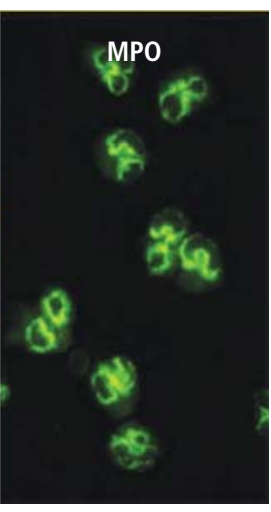

pANCA
FIGURE. Proteinase-3 (PR3)-ANCA and myeloperoxidase (MPO)ANCA are key findings. On ethanol-fixed neutrophils, PR3-ANCA cause a characteristic cytoplasmic granular centrally accentuated immunofluorescence pattern, referred to as CANCA (middle), while MPO-ANCA causes a perinuclear immunofluorescence pattern, referred to as PANCA (right).

Reprinted with permission from Elsevier (Specks U, et al. Anticytoplasmic autoantibodies in the diagnosis and follow-up of Wegener's granulomatosis. Mayo Clin Proc 1989, 64:28-36). Copyright $\odot 1989$ Mayo Foundation for Medical Education and Research.

the analytic sensitivity and specificity of the test. Analytic sensitivity refers to the accurate identification of the presence of ANCA, and analytic specificity refers to measurement of only the entity in question (ANCA), not confounded by the presence of other entities (antibodies).

Equally as important as analytic accuracy is the appropriate ordering of the tests in the right clinical setting. Using a test that is sensitive to the presence of a specific ANCA type accurately identifies the presence of either proteinase-3 (PR3)- or myeloperoxidase (MPO)-ANCA. Once obtained, test results must be evaluated in terms of their relationship to the diagnosis being considered. If the tests are deemed diagnostically useful based on the results, the data can be used to assess the positive and negative predictive value of the tests.

For ANCA-associated vasculitis such as granulomatosis with polyangiitis (GPA, Wegener's granulomatosis), microscopic polyangiitis, and eosinophilic GPA (ChurgStrauss syndrome), PR3-ANCA and MPO-ANCA are key findings. On ethanol-fixed neutrophil staining, PR3ANCA results in a characteristic cytoplasmic granular centrally accentuated staining pattern, referred to as cANCA, while MPO-ANCA causes a perinuclear staining pattern, referred to as pANCA (Figure).

\section{Immunofluorescence or antigen-specific testing- or both?}

A definitive diagnosis is more likely if an immunofluorescence staining pattern of $\mathrm{CANCA}$ is paired with the antigen specificity of PR3-ANCA, for example, or a perinuclear immunofluorescence pattern (pANCA) is paired with a positive MPO-ANCA. When positive test pairings have been obtained and the patient's antigen ANCA reactivity is known, subsequent serial ANCA testing with an antigen-specific assay alone may be indicated, because the ANCA types of patients with vasculitis are unlikely to switch between PR3 and MPO during the course of their disease. If matching pairings are not obtained, the diagnostic utility of the tests remains unconfirmed.

Antigen type (PR3 or MPO) is determined through antigen-specific methods that include solid-phase assays and other methods of bringing the specific antigen in contact with the specific antibody in question. There are two categories of solid-phase assays: the enzyme-linked immunoabsorbent assay (ELISA) and the capture ELISA. In the ELISA methodology, the antigen is directly coated to a plastic plate; in the capture ELISA, an anchor, usually a monoclonal antibody or combination of antibodies, captures the target antigen on the plate. In both ELISA and capture ELISA assays, ANCA contained in the serum sample subjected to testing bind to the immobilized antigen. The amount of ANCA bound to the antigen can then be detected by a secondary antibody that is conjugated with an enzyme that can elicit a color reaction. The intensity of the color reaction is proportional to the amount of ANCA bound to the immobilized antigen.

The ELISA methodology tends to trade off analytic sensitivity for specificity, since the antigen purification process (which allows the ELISA system to increase its specificity) can cause conformational changes to the antigen being bound to the plate. This, in turn, causes a loss of some recognition of the conformationally sensitive ANCA.

In capture ELISA, a specific antibody captures the antigen; this stabilizes the conformation, boosts the analytic sensitivity, and allows a gentler purification process because it only captures the antigen in question and then binds it to the plate. This process decreases false-positive test results caused by residual contaminants in the antigen preparation. Analytic sensitivity issues may come into play if the anchoring monoclonal antibody competes for the epitope on the antigen being recognized by the serum antibody in question (ANCA), causing occasional false-negative results.

Another method now applied to commercial ANCA testing involves bead-based multiplex assays. These assays are based on principles similar to the ELISA or capture ELISA methods. In multiplex microsphere technology, the purified antigen is bound to a polystyrene microsphere instead of a plate. The microsphere is then presented to the antibody in question. The bead is then introduced to a secondary antibody labeled with a fluorescent marker (instead of an enzyme) for detection of the antibody. One advantage of this system is that various beads containing different antigens can be introduced to the same serum sample, and then different color reactions can be measured for each bead. Because only one antigen is bound to each microsphere (eg, PR3-ANCA, MPOANCA or other specific autoantibodies), only specific 


\section{INTERPRETING ANCA RESULTS: Accurate tests, appropriate orders}

Two case histories demonstrate how to analyze conflicting test results.

\section{Case 1: 84-year-old woman with nonspecific interstitial infiltrates}

An 84-year-old woman presented with nonspecific interstitial infiltrates observed on computed tomography. Antineutrophil cytoplasmic antibody (ANCA) testing produced these results:

- cANCA

- pANCA

- myeloperoxidase (MPO)-ANCA

- proteinase 3 (PR3)-ANCA

Negative

Negative

Negative

Positive

The conflicting results, positive PR3-ANCA and negative CANCA, represent a mismatch and raise the question of whether the patient has ANCA-associated vasculitis. Because there is a low pretest probability for CANCA/PR3-ANCA-associated disease in this patient, the positive PR3-ANCA result is questionable. Additional analysis reveals that a new lot of reagents had been associated with false-positive results. Validation of antigen-specific test results using PR3-transfected human mast cell line testing shows absence of PR3 antibodies. The finding indicates that the PR3-ANCA-positive test should be ignored as a methodologic artifact causing an analytic specificity problem.

Comment. Conflicting ANCA-testing results must be reviewed with an understanding of the clinical context, awareness of the assays that provided the results, and availability of an alternative verification method. Positive predictive value of the test result depends not only on the method's accuracy, but also on the appropriate application of the test system. If a test is accurate and the probability of the assumed disease is high, the test result is likely to be reliable. However, if the same accurate test is ordered in a situation where the probability of the disease is low (for example, indiscriminate ordering of tests in low-risk patients), false-negative and false-positive results are more likely despite the accuracy of the test. ${ }^{2}$ This patient had a low pretest probability of CANCA/ PR3-ANCA-associated disease. If the test had been positive for MPO-ANCA-associated disease, the probability would be higher, as some patients with microscopic polyangiitis and MPO-ANCA are found to have pulmonary interstitial infiltrates

antibodies will react to each bead of a specific color. If there is no MPO antibody in the sample, there will be no reaction against the MPO antigen bead; however, if PR3-ANCA is present in the sample, it would react with the PR 3 antigen beads. Using this methodology, a single serum sample can be tested for a multitude of autoantibodies at the same time (see "Interpreting ANCA results: Accurate tests, appropriate orders,"2-10 above). or lung fibrosis at the time of diagnosis. Because the PR3ANCA was positive, however, further analysis was required.

\section{Case 2: 55-year-old man with destructive nasal} disease and hearing problems

A 55-year-old man presented with destructive nasal disease and hearing problems, no systemic symptoms, and no other evidence of vasculitis. Routine testing to rule out limited granulomatosis with polyangiitis (GPA) provided these results:

- pANCA

- MPO-ANCA

Positive

- PR3-ANCA

Negative

Positive

The mismatched pairing of test results indicates a need for further investigation. The patient is shown to have cocaine-induced midline destructive lesions (CIMDL), which are typically positive for elastase antibodies that cause a perinuclear staining pattern on ethanol-fixed neutrophils (pANCA). Coexisting antibodies against multiple antigens can cause positive PR3-ANCA test results. The key test results for CIMDL are: pANCA-positive, MPO-ANCA-negative, human neutrophil elastase (HNE)-ANCA-positive, and possibly PR3-ANCA-positive. ${ }^{3,4}$

Comment. CIMDL clinically mimics GPA, only with more severe local destruction, fewer systemic symptoms, and no other organ involvement. ${ }^{3}$ Generally, ANCA results would be pANCA-positive, MPO-ANCA-negative, possibly PR3ANCA-positive (50\% of the time), and HNE-ANCA-positive. ${ }^{4}$ The HNE- and PR3-ANCA types are not simply crossreacting antibodies, but coexisting separate antibodies. ${ }^{5}$ This phenomenon also occurs in medication-induced ANCA-associated vasculitis, where multiple reactivities are seen with multiple different antigens.

Complicating the CIMDL diagnosis is the increasingly common cocaine contaminant, levamisole, a well-known immunomodulator that has been associated with ear lobe necrosis, skin necrosis and vasculitis (from microthrombotic vasculopathy of the skin), agranulocytosis, antiphospholipid antibodies that possibly play a role in the microthrombotic vasculopathy, and, commonly, ANCA directed against multiple antigens. ${ }^{6-9}$ Levamisole was withdrawn from the US market in $2000 . .^{10}$

\section{WHAT IS THE PROGNOSTIC VALUE OF SERIAL ANCA TESTING?}

Persistent changes in ANCA levels in relapsing disease may have some value in predicting outcome. The issues to consider include the methodology used to determine serial ANCA levels, correlations between ANCA and disease activity, and the use of ANCA changes to guide treatment. 


\section{Does methodology matter when determining serial ANCA levels?}

Methodology in serial ANCA testing is probably unimportant as long as the same method is used serially. Analysis of large groups of ANCA-positive patients show a statistically highly significant correlation among results obtained with different detection methods, including immunofluorescence, direct ELISA, or capture ELISA. However, at the individual patient level there is some variability.

\section{Do ANCA levels correlate with disease activity?}

In a prospective study, serial ANCA samples obtained during the Wegener's Granulomatosis Etanercept Trial $(\mathrm{WGET})^{11}$ were processed in the same manner (collected every 3 months, mean follow-up of 22 months, uniform handling of samples). All samples were analyzed by capture ELISA, and disease activity was measured by the Birmingham Vasculitis Activity Score for Wegener's Granulomatosis (BVAS/WG). The results indicated that an increase in PR3-ANCA levels was not a significant predictor of relapse. The frequency of a relapse within 1 year of an increase in PR3-ANCA levels was found to be approximately $50 \%,{ }^{11}$ a result similar to that reported in several smaller studies of different design and methodology.

\section{Should ANCA changes guide treatment?}

The available data regarding serial ANCA testing are limited mostly to PR3-ANCA. Serial ANCA testing has limited value as a guide to treatment and, in general, changes in ANCA levels alone should not be used to guide treatment decisions. In new patients without documented serial ANCA level associations, an increase in PR3-ANCA levels has no reliable predictive value. The existing literature suggests that this lack of association is not dependent on the method used for ANCA detection. For individual patients in whom long-term serial ANCA testing has been performed and a relationship between PR3-ANCA levels and disease activity has been established, serial ANCA testing can have some predictive value and can be used to guide treatment. For example, when remission is achieved by depleting $\mathrm{B}$ cells in patients with chronically relapsing GPA, ANCA levels usually go down. After B-cell reconstitution, the ANCA level rises in most patients, and this rise is usually associated with a flare shortly thereafter. A flare can be preempted when this pattern is determined in a specific patient, and preemptive treatment is applied accordingly. ${ }^{12}$

\section{WHAT IS THE IMPLICATION OF ANCA TYPE?}

Available reports consistently suggest that PR3-ANCA is associated with a higher mortality than MPO-ANCA (relative risk $[R R], 3.78),{ }^{13}$ and a higher relapse rate. ${ }^{14,15}$ A more rapid loss of renal function among patients with glomerulonephritis and PR3-ANCA than those with
MPO-ANCA has also been reported. ${ }^{16}$ Using remission as the starting point, the number of days from complete remission to first disease flare was plotted for patients with MPO- versus PR3-ANCA in an analysis of longterm data from the Rituximab in ANCA-Associated Vasculitis (RAVE) trial. ${ }^{17}$ The resulting curve demonstrated a divergence in the probability of remaining in remission, confirming that remission maintenance is clearly greater in patients with MPO-ANCA than in patients with PR3-ANCA.

The primary end point of the RAVE trial was remission of disease without the use of prednisone at 6 months. There was little difference in end point achieved based on comparison of diagnosis (microscopic polyangiitis or granulomatosis) or treatment arms (rituximab versus cyclophosphamide); however, an analysis of end point data separating the patients by ANCA type showed that the treatment response to rituximab was superior to that of cyclophosphamide among patients with PR3-ANCA, whereas in patients with MPO-ANCA, there was little difference in response associated with either treatment. Regarding the likelihood of attaining an ANCA-negative status after 6 months, again MPO-ANCA patients showed no difference in frequency on either treatment. Among PR3-ANCA-positive patients, $50 \%$ in the rituximab arm attained ANCA-negative status compared with only $17 \%$ in the cyclophosphamide arm. ${ }^{17}$

\section{SUMMARY}

Diagnostic utility of ANCA testing depends on the methodology and clinical setting. Only cANCA/PR3ANCA and pANCA/MPO-ANCA pairings have positive predictive value for diagnosis of small-vessel vasculitis. Mismatches in results, findings of human neutrophil elastase-ANCA, or identification of multiple positive antigens should be considered in cases of cocaine or drug use.

The clinical utility of serial ANCA testing is unconfirmed. Good data currently exist only for PR3-ANCA, and different drugs may affect ANCA levels in different ways. ANCA type is significant in that PR3-ANCA portends a higher relapse rate and poorer patient outcomes compared with MPO-ANCA.

\section{REFERENCES}

1. Russell KA, Wiegert E, Schroeder DR, Homburger HA, Specks U. Detection of anti-neutrophil cytoplasmic antibodies under actual clinical testing conditions. Clin Immunol 2002; 103:196-203.

2. Langford CA. The diagnostic utility of c-ANCA in Wegener's granulomatosis. Cleve Clin J Med 1998; 65:135-140.

3. Trimarchi M, Gregorini G, Facchetti F, et al. Cocaine-induced midline destructive lesions: clinical, radiographic, histopathologic, and serologic features and their differentiation from Wegener granulomatosis. Medicine 2001; 80:391-404.

4. Wiesner O, Russell KA, Lee AS, et al. Antineutrophil cytoplasmic antibodies reacting with human neutrophil elastase as a diagnostic marker for cocaine-induced midline destructive lesions but not autoimmune vasculitis. Arthritis Rheum 2004; 50:2954-2965. 
5. Peikert T, Finkielman JD, Hummel AM, et al. Functional characterization of antineutrophil cytoplasmic antibodies in patients with cocaine-induced midline destructive lesions. Arthritis Rheum 2008; 58:1546-1551.

6. Knowles L, Buxton JA, Skuridina N, et al. Levamisole tainted cocaine causing severe neutropenia in Alberta and British Columbia. Harm Reduct J 2009; 6 (Nov 17):30. doi:10.1186/1477-7517-6-30.

7. Zhu NY, LeGatt DF, Turner AR. Agranulocytosis after consumption of cocaine adulterated with levamisole. Ann Intern Med 2009; 150:287-289.

8. Bradford M, Rosenberg B, Moreno J, Dumyati G. Bilateral necrosis of earlobes and cheeks: another complication of cocaine contaminated with levamisole. Ann Intern Med 2010; 152:758-759.

9. Waller JM, Feramisco JD, Alberta-Wszolek L, McCalmont TH, Fox LP. Cocaine-associated retiform purpura and neutropenia: is levamisole the culprit [published online ahead of print March 20, 2010]? J Am Acad Dermatol 2010; 63:530-535. doi: 10.1016/j. jaad.2010.01.055

10. Chang A, Osterloh J, Thomas J. Levamisole: a dangerous new cocaine adulterant [published online ahead of print July 28, 2010]. Clin Pharmacol Ther 2010; 88:408-411. doi:10.1038/clpt.2010.156

11. Finkielman JD, Merkel PA, Schroeder D, et al. Antiproteinase 3 antineutrophil cytoplasmic antibodies and disease activity in Wegener granulomatosis. Ann Intern Med 2007; 147:611-619.

12. Cartin-Ceba R, Golbin J, Keogh KA, et al. Rituximab for remis- sion induction and maintenance in granulomatosis with polyangiitis (Wegener's): a single-center ten-year experience [published online ahead of print June 21, 2012]. Arthritis Rheum. doi: 10.1002/art.34584

13. Hogan SL, Nachman PH, Wilkman AS, Jennette JC, Falk RJ; and the Glomerular Disease Collaborative Network. Prognostic markers in patients with antineutrophil cytoplasmic autoantibodyassociated microscopic polyangiitis and glomerulonephritis. J Am Soc Nephrol 1996; 7:23-32.

14. Booth AD, Almond MK, Burns A, et al. Outcome of ANCAassociated renal vasculitis: a 5-year retrospective study. Am J Kidney Dis 2003; 41:776-784.

15. Jayne D, Rasmussen N, Andrassy K, et al. A randomized trial of maintenance therapy for vasculitis associated with antineutrophil cytoplasmic autoantibodies. N Engl J Med 2003; 349:36-44.

16. Franssen CFM, Gans ROB, Arends B, et al. Differences between anti-myeloperoxidase- and anti-proteinase 3-associated renal disease. Kidney Int 1995; 47:193-199.

17. Stone JH, Merkel PA, Spiera R, et al; for the RAVE-ITN Research Group. Rituximab versus cyclophosphamide for ANCAassociated vasculitis. N Engl J Med 2010; 363:221-232.

Correspondence: Ulrich Specks, MD, Division of Pulmonary and Critical Care Medicine, Mayo Clinic College of Medicine, 200 First St. SW, Rochester, MN 45905; specks.ulrich@mayo.edu

\title{
Defining disease activity and damage in patients with small-vessel vasculitis
}

\author{
PETER A. MERKEL, MD, MPH \\ Chief, Division of Rheumatology, Professor of Medicine and Epidemiology, University of Pennsylvania, Philadelphia, PA
}

\section{ABSTRACT}

The complexity of small-vessel vasculitis requires repeated evaluations of disease activity and damage. Clinical assessment, including regular restaging of disease, is important for management of therapeutic interventions; similarly, assessment tools must be standardized and validated for use in the clinical trial setting. The Outcome Measures in Rheumatology group promotes validated outcomes measures for use in trials. Validated tools for use in clinical trials include the Birmingham Vasculitis Activity Score and the Vasculitis Damage Index. In addition, health-related quality of life assessments underscore the importance of patient-ranked issues in assessing and treating vasculitis. Improvements in the clinical treatment of vasculitis will arise from research that is supported by refined and validated assessment tools.

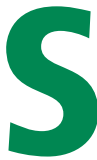

mall-vessel vasculitides are complex diseases with highly variable clinical features and are associated with considerable morbidity and mortality. These systemic, multisystem, multiorgan diseases often threaten vital organs with manifestations that include upper airway disease, pulmonary disease, glomerulonephritis, neuropathy, arthritis/arthralgias, malaise/fatigue, eye disease, skin/mucosa irregularities, vascular disease, cardiac disease, and gastrointestinal disease.

Accurate assessment of the patient with vasculitis is a challenge for the clinician and is critical for managing therapeutic interventions throughout the course of the disease. Effective management includes repeated evaluations of the activity and severity of the disease as well as the damage it has caused. These distinct yet overlapping concepts must be measured separately but evaluated as a whole. Additional categorizations of disease course,
Dr. Merkel reported that he has served as a consultant for Actelion Pharmaceuticals US, Inc.; Avila Therapeutics; EMD Serono, Inc.; Human Genome Sciences, Inc.; and Nordic Pharma Group and has received research support from Actelion Pharmaceuticals US, Inc.; Bristol-Myers Squibb; CaridianBCT, Inc.; Celgene Corporation; Genentech, Inc.; and Medlmmune, LLC.

This article was developed from an audio transcript of Dr. Merkel's presentation at the "New Directions in Small-Vessel Vasculitis: ANCA, Target Organs, Treatment, and Beyond" symposium held at Cleveland Clinic on May 4, 2011. The transcript was formatted and edited by Cleveland Clinic Journal of Medicine staff for clarity and conciseness, and was then reviewed, revised, and approved by Dr. Merkel.

doi:10.3949/ccjm.79.s3.03 
such as whether it is active (new-onset, persistent, or flare) or in remission, further define the disease and are routinely employed in guiding treatment choices.

The importance of accurately assessing a patient's clinical status is clear, but it is also important to standardize and quantify vasculitis assessment tools for use in clinical trials. Standardized assessments are needed to:

- Guide clinical trial enrollment criteria

- Describe and compare study populations

- Quantify and measure treatment effectiveness

- Describe long-term outcomes

- Translate standardized assessment tools into clinical practice.

Over the past few decades, improvements in clinical research have resulted in increasingly accurate data obtained from well-designed randomized controlled trials, all of which are based on better clinical assessments. Improving the quality of the assessment tools has improved both the interpretation of trial results and translation of findings into clinical practice.

\section{DISEASE ASSESSMENT}

When assessing patients with vasculitis, whether clinically or in the context of a clinical trial, it is essential to differentiate among disease activity, damage, and severity:

Disease activity, such as active bleeding or mucosal inflammation, is treatable and potentially responsive to therapy.

Disease damage is generally irreversible and not improved by treating vasculitis. Damage may be caused by the disease itself, its treatment, or a comorbid condition. In general, once damage is identified, it is considered permanent if it remains unchanged for more than 6 months. In the Wegener's Granulomatosis Etanercept Trial, ${ }^{1}$ damage that occurred in more than $10 \%$ of the cohort included hearing loss; proteinuria $(\geq 0.5 \mathrm{~g} / 24$ hours); nasal blockage, chronic discharge, or crusting; nasal bridge collapse or septal perforation; glomerular filtration rate at least 50\% lower than premorbid baseline; subglottic stenosis; and chronic sinusitis or radiologic damage. Disease-related damage can be addressed; a saddle-nose deformity can respond to plastic surgery, for example, but treating vasculitis will have no effect on the underlying established anatomic defect.

Disease severity assesses the intensity of the disease and guides the clinician in gauging how aggressive the therapy should be.

Vasculitis has two primary disease states: remission and active disease. In remission, there is no evidence of active disease. This is often qualified by describing the remission as either complete or partial; it is further defined by introducing an element of time, such as a "sustained" remission of more than 6 months. Active disease is the presence of any ongoing expression of vasculitis that is not caused by disease damage, comorbidity, or treatment. Active disease can be graded as low, medium, or high; if active disease lasts longer than 6 months, it is described as persistent or sustained. Flare, a manifestation of active disease, describes the transition from remission to active disease and is characterized by worsening of disease activity. Flares are graded as nonsevere or severe.

These descriptions of disease status can be further broken down into whether they are occurring "on" or "off" treatment. All of these elements are important and the subtleties and differences are critical in interpreting data for use in the clinical setting or in clinical trials.

\section{CLINICAL ASSESSMENT}

Assessing the status of disease for a patient with granulomatosis with polyangiitis (GPA, Wegener's granulomatosis [WG]) or microscopic polyangiitis (MPA) begins with a detailed medical history and physical examination every time the patient is seen. Appropriate laboratory assessments include a complete blood count, tests of renal function, acute phase reactants (possibly as disease markers, but not necessarily to guide therapy), and other laboratory tests as needed. Controversy exists regarding the role of antineutrophil cytoplasmic antibody (ANCA) testing in assessment of disease activity.

Urinalyses are key for assessing activity; if a urine dipstick result is positive, a subsequent microscopic examination should be conducted. Microscopic review may demonstrate red cell casts that a routine laboratory check may not reveal. In addition to spotting de novo hematuria, looking for a change in dipstick results may prove valuable, since hematuria may increase in patients in whom persistent hematuria has already been noted. The change may be due to renal disease from the vasculitis, cyclophosphamide-induced bladder toxicity, a kidney stone, menses, or a variety of other causes, but if the hematuria is not monitored, a key assessment will be missed.

Disease staging through diagnostic imaging of the sinuses, neck, and chest should be performed on a regular basis as appropriate, beginning at the patient's initial visit. Restaging, in much the same way as an oncologist restages cancer, should take place regularly, because this informs whether to make a major change in therapy (eg, from cyclophosphamide, azathioprine, or rituximab). Restaging will also allow benchmarking of old, new, and changed damage so that when the disease recurs, the existing damage can be differentiated from new lesions. Once the disease has stabilized, imaging can be discontinued.

Consultations with otolaryngologists, ophthalmologists, cardiologists, and other specialists should be sought as needed. Serial audiograms, laryngoscopy, echocardiograms, and other appropriate tests should be performed as required. Biopsies are useful for assessment of patients, particularly at diagnosis, but also when it becomes necessary to reassess the progress of a patient's disease or to identify a potential infection versus a possible malignancy. Biopsy is particularly helpful for kidney disease. If 


\section{TABLE}

\section{Commonly used outcome measures for vasculitis}

Disease activity

Birmingham Vasculitis Activity Score (BVAS) 2,10

BVAS/Wegener's granulomatosis (BVAS/WG) ${ }^{3,10}$

BVAS version 3 , 10

Physician's global assessment ${ }^{\mathrm{a}}$

Biomarkers $^{\mathrm{a}}$ (antineutrophil cytoplasmic antibodies [ANCA], acute-phase reactants)

\section{Disease damage}

Vasculitis Damage Index ${ }^{5}$

ANCA-Associated Vasculitis Index of Damage ${ }^{6}$

Combined Damage Assessment Index ${ }^{7}$

End-stage renal disease

Death

\section{Prognosis}

Disease Extent Index 8,10

Five-Factor Score ${ }^{9,10}$

ANCA type

Disease type

Flare history

Health-related quality of life/patient-reported outcomes

Medical Outcomes Study 36-item short-form health survey

Patient Global Impression of Change

${ }^{\mathrm{a}}$ Not well validated, controversial

kidney function is deteriorating without other evidence of active disease, then repeat biopsy is appropriate to determine whether the deterioration is associated with persistent active disease, the natural history of declining kidney function, or another cause. Patients with vasculitis may develop new comorbidities, particularly infections, so vigilance is always required. Importantly, documentation and awareness of disease-related damage are crucial in order to avoid overtreatment; damage should not be treated if therapy will not improve it.

\section{ASSESSING DISEASE ACTIVITY AND DAMAGE}

In the clinical trial setting, GPA and MPA are assessed using the outcomes measures listed in the Table. ${ }^{2-10}$

\section{Birmingham Vasculitis Activity Score}

Introduced in 1994, the Birmingham Vasculitis Activity Score (BVAS) is a single-page checklist that records weighted data on more than 50 items and nine organ systems; the sum of the individual items provides the

\section{Assessment with the BVAS/WG}

Patient 1 presents with fatigue, fever, purpura, arthritis, rhinitis, sinusitis, conductive hearing loss, and pulmonary nodules; patient 2 presents with alveolar hemorrhage and fever. Patient 1 has active disease and a BVAS/WG of 8 . Patient 2 has active disease and a BVAS/WG of 4 .

Both patients require attention, but, despite the lower score, Patient 2 warrants more immediate attention because of the alveolar hemorrhage and in that sense may be considered "sicker."

Which patient is more likely to enter a sustained clinical remission? Patient 1's phenotype increases the likelihood of relapse while sustained remission is more likely for Patient 2.

BWAS/WG = Birmingham Vasculitis Ativity Score/Wegener's Granulomatosis ${ }^{3}$

final score. ${ }^{2}$ There have been two revisions of the BVAS; one focuses on GPA (BVAS/WG) ${ }^{3}$ and the other, BVAS version 3 (v.3) is more simplified. ${ }^{4}$ For all of the BVAS tools, remission is defined as a score of 0 . Any score greater than 0 defines active disease. Each system is evaluated as being active or not, with items characterized as more severe being weighted more heavily. The use of the BVAS/WG is illustrated in two patients (see "Assessment with the BVAS/WG," above).

Each of the three BVAS tools has advantages and disadvantages. All of the tools are validated and fairly easy to use; they are inexpensive; they have been employed successfully in clinical trials; and the results are widely accepted by investigators, industry, and both the US Food and Drug Administration (FDA) and the European Medicines Agency. The tools miss some variables, however, including biomarkers and the patient's own input; it takes training to learn how to use the tools; decisions are subjective, because the investigator must decide whether the disease is active; because the tools lack gradation, a listing of hemorrhage, for example, does not consider the degree of severity of the hemorrhage; weighting is potentially inaccurate and open to interpretation; precision and sensitivity are inadequate; and there are multiple versions, although they have been shown to be well correlated. ${ }^{10}$

Every major randomized controlled trial in the past 15 years has used the BVAS or one of its derivatives to define outcomes, but primary outcomes were not defined strictly from the BVAS itself. There were important differences in the trials' definitions of remission, which is the outcome of interest. For example, some trials allow for minor disease activity concurrent with partial remission, while others require full absence of disease activity to achieve "remission." 


\section{Vasculitis Damage Index}

The Vasculitis Damage Index (VDI) is a single-page catalog of damage items separated into 11 groupings. Limitations of the index include lack of attribution (to vasculitis, treatment, or comorbidities), gradation, weighting, and patient input (patient-reported outcomes). ${ }^{5}$ Revisions to the VDI have been made in the ANCA Vasculitis Index of Damage (AVID) ${ }^{6}$ which incorporates an expanded list of damage items, as well as an even more expanded version called the Combined Damage Assessment Index that combines the items from the VDI and AVID. ${ }^{7}$ While these tools provide a means to catalog damage by choosing whether an item is present or not, a more data-driven approach to damage assessment is needed that incorporates weighting into the tool.

Damage assessment may be the most important measure in evaluating the patient with vasculitis. In addition to keeping patients alive, one of the main purposes in treating active disease is to prevent damage, maintaining quality of life for the patient for the long term and improving outcomes.

\section{PATIENT-REPORTED OUTCOMES}

Patients have a different perspective on their disease than that provided by assessment tools or physicians. Because physician and patient ratings are often disparate, health-related quality of life (HRQOL) is an increasingly important outcome measure for patients as well as regulatory agencies. In a 2010 study, structured patient-reported assessments of burden of disease were obtained from 264 patients with vasculitis in the United States, Germany, and the United Kingdom. Patients ranked items in terms of most frequent burdens of disease. Across ages and countries, patients most commonly rated fatigue/energy loss, pain, musculoskeletal symptoms, and social manifestations as the most severe ramifications of their disease. ${ }^{11}$ None of the burdens of disease identified in this study are universally measured in the current assessment tools. Patients with active disease had more of the most commonly listed burden-ofdisease items; however, patients still suffered from these burdens when the disease was inactive. These disease burden items are therefore mostly dynamic problems and not simply chronic issues.

Patient ratings differ considerably from physician ratings in terms of importance. For example, patients rate nasal manifestations, weight gain, and some chronic pain and fatigue items higher than renal insufficiency and stroke. There is a clear need to address not only physician-ranked issues, but also patient-ranked issues in assessing and treating vasculitis. ${ }^{11}$

When measuring HRQOL via the Medical Outcomes Study 36-item short-form health survey (SF-36) in patients with vasculitis, a correlation is noted between QOL and sustained remission. In a study by Tomasson et al, QOL was measured using the SF-36 upon treatment following a flare. ${ }^{12}$ In all patients, SF-36 increased dramatically immediately following treatment but then leveled off over time. In patients who achieved sustained remission, SF-36 scores continued to rise from baseline. In patients who did not achieve a sustained remission, the SF-36 scores did not improve. This QOL measure, therefore, captures a value that other assessments do not, further demonstrating its utility as part of the assessment process.

\section{VALIDATED OUTCOME MEASURES}

Outcome Measures in Rheumatology (OMERACT) is an international group of clinicians, trialists, epidemiologists, biostatisticians, health economists, industry executives, and FDA and European Medicines Agency officials who meet every 2 years to promote data-based validation of outcome measures for a variety of diseases. OMERACT endorses core sets of validated outcomes when data demonstrate veracity, discrimination, and feasibility. ${ }^{13}$ For each domain in the vasculitis arena, there is an associated validated instrument: for disease activity, the validated instruments are the BVAS, BVAS/WG, and BVAS v.3; for damage assessment, the instrument is the VDI; for patient-reported outcomes, the instrument is the SF-36; and finally, for mortality, the instrument is death. ${ }^{13}$ This core set of measures helps frame how future trials in vasculitis will be standardized and assists in comparing trials, which is particularly important to regulatory agencies.

The tools for disease assessment in vasculitis still need to be refined for activity and damage assessment in order to be more scalable and precise, thereby measuring smaller effects. Patient-reported outcomes and patient perspectives on disease need to be better captured, and reliable biomarkers need to be discovered or further developed. Improved outcome measures must be developed for other types of vasculitis, such as eosinophilic granulomatosis with polyangiitis (Churg-Strauss syndrome), giant cell (temporal) arteritis, and Takayasu arteritis,${ }^{14}$ in order to conduct and report trial results. These outcome measures could also translate into tools that can be used to assess patients and make treatment decisions, thereby helping the clinician at the bedside.

\section{REFERENCES}

1. Seo P, Min YI, Holbrook JT, et al. Damage caused by Wegener's granulomatosis and its treatment: prospective data from the Wegener's Granulomatosis Etanercept Trial (WGET). Arthritis Rheum 2005; 52:2168-2178.

2. Luqmani RA, Bacon PA, Moots, RJ, et al. Birmingham Vasculitis Activity Score (BVAS) in systemic necrotizing vasculitis. QJM 1994;87:671-678.

3. Stone JH, Hoffman GS, Merkel PA, et al. A disease-specific activity index for Wegener's granulomatosis: modification of the Birmingham Vasculitis Activity Score. International Network for the Study of the Systemic Vasculitides (INSSYS). Arthritis Rheum 2001; 44:912-920.

4. Mukhtyar C, Lee R, Brown D, et al. Modification and validation 
of the Birmingham Vasculitis Activity Score (version 3). Ann Rheum Dis 2009; 68:1827-1832.

5. Exley AR, Bacon PA, Luqmani RA, et al. Development and initial validation of the Vasculitis Damage Index for the standardized clinical assessment of damage in the systemic vasculitides. Arthritis Rheum 1997; 40:371-380.

6. Seo P, Luqmani RA, Flossmann O, et al. The future of damage assessment in vasculitis. J Rheumatol 2007; 34:1357-1371.

7. Seo P, Jayne D, Luqmani R, Merkel PA. Assessment of damage in vasculitis: expert ratings of damage. Rheumatology (Oxford) 2009; 48:823-827.

8. de Groot K, Gross WL, Herlyn K, Reinhold-Keller E. Development and validation of a disease extent index for Wegener's granulomatosis. Clin Nephrol 2001; 55:31-38.

9. Guillevin L, Pagnoux C, Seror R, Mahr A, Mouthon L, Le Toumelin P; French Vasculitis Study Group (FVSG). The Five-Factor Score revisited: assessment of prognoses of systemic necrotizing vasculitides based on the French Vasculitis Study Group (FVSG) cohort. Medicine (Baltimore) 2011; 90:19-27.

10. Merkel PA, Cuthbertson DD, Hellmich B, et al. Comparison of dis- ease activity measures for anti-neutrophil cytoplasmic autoantibody (ANCA)-associated vasculitis. Ann Rheum Dis 2009; 68:103-106.

11. Herlyn K, Hellmich B, Seo P, Merkel PA. Patient-reported outcome assessment in vasculitis may provide important data and a unique perspective. Arthritis Care Res (Hoboken) 2010; 62:1639-1645.

12. Tomasson G, Boers M, Walsh M, et al. Assessment of health related quality of life as an outcome measure in granulomatosis with polyangiitis (Wegener's). Arthritis Care Res (Hoboken) 2012; 64:273-279.

13. Merkel PA, Aydin SZ, Boers M, et al. The OMERACT core set of outcome measures for use in clinical trials of ANCA-associated vasculitis. J Rheumatol 2011; 38:1480-1486.

14. Direskeneli H, Aydin SZ, Kermani TA, et al. Development of outcome measures for large-vessel vasculitis for use in clinical trials: opportunities, challenges, and research agenda. J Rheumatol 2011; 38:1471-1479.

Correspondence: Peter A. Merkel, MD, MPH, Chief, Division of Rheumatology, University of Pennsylvania, 8th Floor Penn Tower, 3400 Spruce Street, Philadelphia, PA 19104; pmerke/@upenn.edu 